\title{
The Research of the Design of 3D Information Gene Model Oriented to Machine Vision
}

\author{
Yijie Hao $^{1}$, Shun Zhang ${ }^{2, *}$ and Chunfeng Shen ${ }^{3}$ \\ ${ }^{1}$ Rabun Gap-Nacoochee School, Shanghai 200092, China \\ ${ }^{2}$ The CAD Research Center, Tongji University, Shanghai 200092, China \\ ${ }^{3}$ Shanghai Baosight Software Co., Ltd, Shanghai 200000, China \\ ${ }^{*}$ Corresponding author
}

\begin{abstract}
This paper introduces the concept of genes to the design of the product, put forward and set up product genetic model, aimed at apply the principle of gene expression to implement the product intelligent design, and strengthen the autonomy of product design, reduce artificial participation and related investment, improve the efficiency of product design, promote the development of product intelligent design. This paper first analyzes the problems existing in products genetic model, based on this, puts forward and establish the genetic model based on the feature of the products. On the basis of the definition of product gene structure, puts forward binary coding system of product gene and related operations. Then, based on product gene model, establish gene expression process model, product gene expression process is divided into two steps, transcription and translation, and transcription can be divided to two stages respectively. Finally, apply the theory of this paper to the engine products, to implement the intelligent design of the engine product, and has obtained the expected effect.
\end{abstract}

Keywords-component; feature; product gene; gene coding; gene operation; gene expression

\section{INTRODUCTION}

With the development of modern industry, the product design process has become increasingly complex and sophisticated, intelligent design modeling has become the inevitable trend of product design modeling. In recent years, research on product gene focused on two directions. First, establishment and study about concepts and models of product gene; Second, study on acquisition, restructuring and expression technology of product gene. Holland proposed gene model on the basis of explaining the evolution and design activities of biological analogies; Richard proposed a gene empirical mode about product conceptual design using genetic algorithm; Chen Yong and others used product characteristics for products' features expression and achieved definition of gene product; Hao Yong-tao and others he established product gene coding scheme by building feature function model of the product ; Li Hongjie made the design of the products' function structure as the core , using functional matrix to make digital and formal descriptions for their needs and have a unified expression for the functional gene ; Zhang Xiangjun and Gui Changlin analyzed the level of the intelligent design and studied the intellectual model of that ; Zhang Zhiwei and others studied a genetic system design model based on the knowledge of gene design to standardize the design process and design operations. These studies focused on the gene product concept and model definition. Although the focus is different, but all of them plays an important role for the modeling of gene products.

Comprehensive analysis shows that the study of gene products has made certain progress, but still exist the following problems:

Acquisition, reorganization, and expression of product gene is the key technology of product evolutionary design, which is the basis of genetic information coding issues. At present a lot of research is the encoding system which based on product features, product function, there is no unified standard. Different ways of encoding has great influence for subsequent genetic recombination, mutation, so must to choose effective and reasonable gene encoding way.

\section{FEATURE DESCRIPTION}

\section{A. The Definition of Feature}

At present, the "characteristics", or "based on the characteristics" these terms appeared frequently in the field of $\mathrm{CAD}$, in creating a product model, it is generally believed that this is a more direct, more useful way to create and expression. Feature as a high-level design concept, containing a large number of designers design intent, is of great significance for the modification of design, maintenance and the subsequent analysis comprehensive process, has a very important role in improve the automation level of CAD system, promote the integration of CAD, CAM and CAPP. Since the 1980s, many scholars have to define feature. C. hayes and P. Wright define the feature of "be continuous process to remove material shape". S.C. Luby and J.K. Dixon defined feature as "a geometric entity, the entity is related with one or more function in CIMS". Dixon pointed out that "feature is the entity with the double attribute of shape and function".

Because in different areas and specific application, feature have different meaning and expression form, thus the concept of feature has many different explanation and definition. Broadly, feature is an integration of object, containing a particular set of design and manufacture connotation of the information. In particular, feature is a specific shape of a set of geometric entity which has topological relationships and its attributes; it contains the abundant engineering semantics, 
expressed product function and shape in a higher level, and closely linked together with industrial manufacturing process.

Taken together, features include the following meanings:

(1) the feature is not the complete parts;

(2) the feature associated with geometric description;

(3) features in the service of information integration among multiple applications;

(4) the form and connotation of the feature is related to specific engineering application;

(5) the feature can be identify, describe and mapping or conversion.

\section{B. The Classification of the Feature}

In a part or product, there are many features which have mutual connection and restriction; they belong to different feature class. Because of the different application, people also have differences classification to feature. According to the application protocol AP214 in STEP standard, features can be divided into seven kinds, such as transition, composite slab reinforcement, combined entity, processing plate reinforcement, general characteristics, distribution characteristics, processing entity class[37]. Paper divided part feature into bearing feature, the shape feature, precision feature and technical feature. In the literature, for CAD/CAPP integration application, divided product features into five categories, such as form feature, material feature, precision feature, process feature, the manufacturing resources features. Literature[39] put forward feature classification in the collaborative design environment and development system, including the overall features, collaboration features, design feature, manufacturing feature and shape feature, technology feature, precision feature and material feature eight classes. Literature[38] put forward the general feature concept face to the product life cycle, divided feature into user feature, functional feature, material feature, shape features and other 16 typical feature.

According to that feature play different role in the process of the parts or product design, this paper divided feature into benchmark, generate, modification, composite feature and technology feature. The benchmark feature refers to the reference point, line, face and coordinate system used to aid other features; Generated features on the benchmark, is the foundation of modified feature and composite feature, it includes stretching, rotation and scanning feature; Modified features is on the basis of benchmark feature, including the round corner, chamfer, holes, pump shell, draft, rib and slot feature; Composite features is the new features which is applying some basic features and modify operations to the basic characteristics, they are often appear on the parts meeting with some fixed relationship, or is the combination of certain kinds of feature, we can regard these features as a feature to analyze artificially, such as array feature, such as strengthening rib feature and mixing feature; Process features including surface roughness, machining precision grades and tolerance and other information.

Of course, the same feature class can also have different division standard. If according to the position relationship between features, we can divide the features into the parent and child. For example, in some parts, there may be other features exist in some feature, such as tensile body or a body of revolution with chamfer, hole and slot, and other features. We can regard the features which load-bearing other features as the father; the other called the child.

\section{Product Gene ModeL}

\section{A. The Definition of Product Gene}

Genes are fragments of function on the DNA molecule, is the basic unit of the genetic information, decided a variety of biological traits; Genes determine the person's physical, intertwined with healthy, beautiful, long life, it is the manipulator and regulators of life. Therefore, where there is life, there is a gene, all the forms of the existence of life and decay are determined by genes, including people's height, appearance, weight, color, character and gene are inseparable.

Gene molecular structure is composed of nucleotides, a molecular deoxyribonucleotide including 1 phosphoric acid, molecular ribodesose and 1 molecules nitrogenous. Nucleotides form a single DNA in the form of sugar phosphate - sugar covalent. Genes through replication can pass on to their offspring, through transcription and translation can guide the protein synthesis, and determine the character of the life. The structure of the DNA molecule as shown.

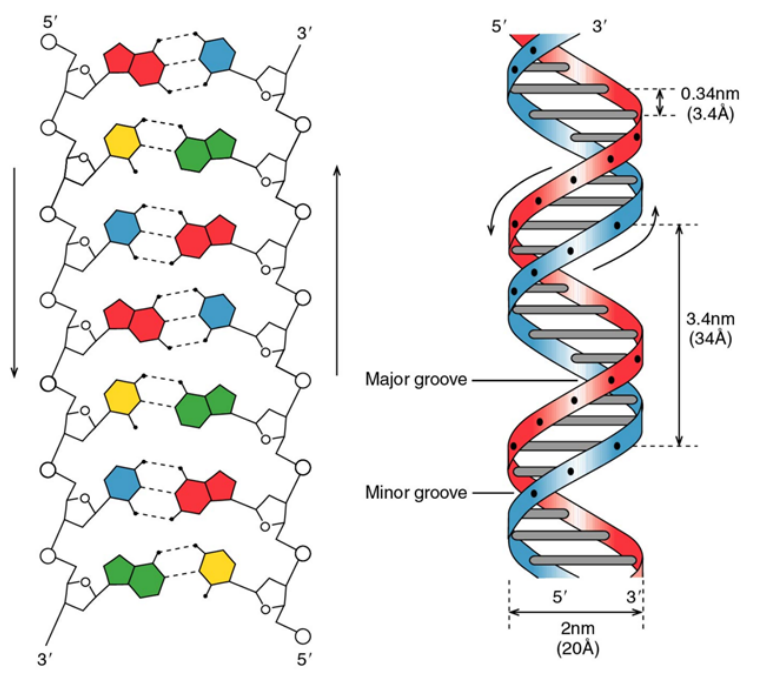

FIGURE I. THE STRUCTURE OF THE DNA

\section{B. Product Gene Encoding}

1) feature-type represent the type of feature, including convex platform, grooves, the body of revolution, scanning body hole, round corner, chamfer, and the pump shell, coding in 1-8. Their code corresponding to the table 1 .

TABLE I. THE CODE OF FEATURE TYPE

\begin{tabular}{|c|c|c|c|c|c|}
\hline feature & $\begin{array}{c}\text { convex } \\
\text { platform }\end{array}$ & groove & $\begin{array}{c}\text { Body of } \\
\text { revolution }\end{array}$ & $\begin{array}{c}\text { Scanning } \\
\text { body }\end{array}$ & $\begin{array}{c}\text { Rounded } \\
\text { corners }\end{array}$ \\
\hline code & 1 & 2 & 3 & 4 & 5 \\
\hline
\end{tabular}


Profile-shape corresponding to the sketch contour shape of feature, can be divided into two types, regular shape and irregular shape. For regular shape, only considers point, line, triangle, rectangle, round (including circular, elliptic), and irregular shape as a general shape, expressed in 1 to 6 respectively. Their code corresponding to the table 2 .

TABLE II. THE CODE OF FEATURE TYPE

\begin{tabular}{|c|c|c|c|c|c|}
\hline $\begin{array}{c}\text { Sketch } \\
\text { shape }\end{array}$ & point & line & triangle & rectangle & round \\
\hline coding & 1 & 2 & 3 & 4 & 5 \\
\hline
\end{tabular}

\section{Product Gene Related Operations}

Biological gene operation including extraction, gene amplification, gene recombination, etc. In this article, the product gene also has a variety of related operations. Based on the biological genetic engineering, puts forward products genetic engineering, to make it easier to express the product gene transcription, translation, recombination and mutation, etc, this study defines a variety of specific operating norms, namely, atomic operation. Mainly divided into the following kinds:

(1) Product gene separation. Assumes that the product gene sequences $\quad G \_s=G \_0 \cdot G \_1 \cdot G \_2 \ldots . . \cdot$ G_n, among them $\mathrm{G} \_\mathrm{i}=\mathrm{g} \_0 \cdot \mathrm{g} \_1 \cdot \mathrm{g} \_2 . . . \cdot \mathrm{g}$ _n, $\mathrm{i} \in \mathrm{N}$, in sequence $\mathrm{G} \_\mathrm{s}$, to produce two new sequence $G \_s 1=G \_0 \cdot G \_1 \cdot G \_2 \ldots . . . G \_j$, G_s2 $=G \_(j+1) \cdot G \_(j+2) \cdot G \_(j+3) \ldots . . . \cdot G \_n$. In this paper, defines the atomic operations S_g.

(2) Product gene combinations. Suppose there are two product gene sequence $\quad G \_s 1=G \_0 \cdot G \_1 \cdot G \_2 \ldots . . \cdot G \_n$, G_s2 $=G \_\left(0^{\wedge^{\prime}}\right) \cdot G \_\left(1^{\wedge^{\prime}}\right) \cdot G_{-}\left(2^{\wedge^{\prime}}\right) \ldots . . . \cdot G_{-}\left(n^{\wedge^{\prime}}\right)$, the first group of genes connected to the second group of genes, and do not change the relative position of two sets of product gene sequences internal gene, form the new gene sequence which has the new function. In this paper, defines the atomic operations C_g.

(3) Product gene cross exchange. Suppose there are two product gene sequence $G \_s 1=G \_0 \cdot G \_1 \cdot G \_2 \ldots . . \cdot G \_n$, G_s2 $=G \_\left(0 \wedge^{\prime}\right) \cdot G_{-}\left(1 \wedge^{\wedge^{\prime}}\right) \cdot G_{-}\left(2^{\wedge^{\prime}}\right) \ldots . . \cdot G_{-}\left(n^{\wedge^{\prime}}\right)$, suppose that cross exchange occur in the jth allele gene, G_s1=G_0 $\cdot G \_1 \cdot G \_2 \ldots . . \cdot G \_(j-1) \cdot G \_\left(j^{\wedge^{\prime}}\right) \ldots . . \cdot G_{-}\left(n^{\wedge^{\prime}}\right)$, $G_{-} s 2=G_{-}\left(0^{\wedge^{\prime}}\right) \cdot G_{-}\left(1^{\wedge^{\prime}}\right) \cdot G_{-}\left(2^{\wedge^{\prime}}\right) \ldots . . \cdot G_{-}\left(\bar{\zeta} j-1 \sum\right.$ $\left.\wedge^{\prime}\right) \cdot G \_j \ldots . . . G \_n$. In this paper, defines the atomic operations E_g.

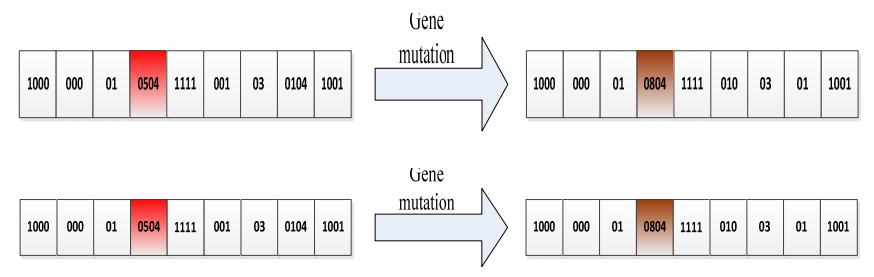

\section{Product Gene Expression}

In biology, the gene expression is mainly by genes control related protein synthesis, and this process can be divided into "transcription" and "translate" two steps. Transcription refers to the double-stranded DNA break down first, and then one of the chain as a template, according to the principle of complementary base pairing, formation of messenger RNA; translation refers to messenger RNA as template, the process of related proteins.

Similarly, the product gene expression process are also divided into "transcription" and "translate" two steps. Gene transcription process is from gene encoding into relational matrix, and then converted to the feature tree; the translation of product gene is the mapping process from the feature tree to product entity structure.

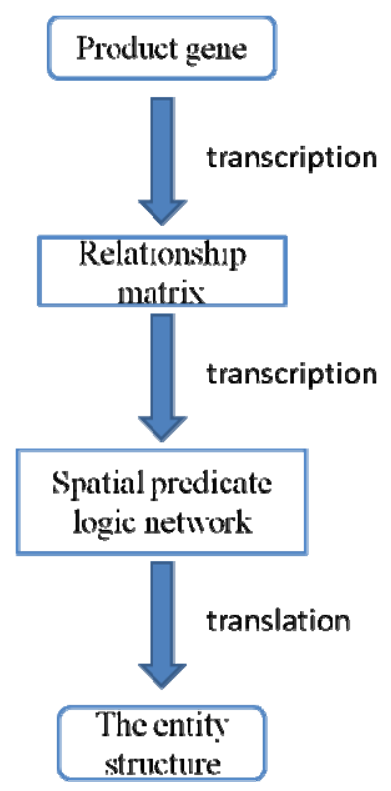

FIGURE II. PRODUCT GENE EXPRESSION PROCESS

In biology, gene transcription is refers to the following process: occurs in the nucleus, with one of the chain of doublestranded DNA structure as a template, based on the principle of complementary base pairing, the process to compound messenger RNA.

In products genetic engineering, gene transcription are divided into two processes, the first process is from product genes to the relational matrix conversion process; the second process is from a relational matrix conversion to the spatial predicate logic network process.

Product gene to the relational matrix transformation rules are as follows:

1) Extract the number of feature gene in product gene, set the matrix size as the maximum number;

2) Set $a \_i j=k, j=i+1 ; \quad a \_i j=-k, i=j+1 ; k$ is spatial predicate logic code for feature $\mathrm{j}$, other a_ij=0;

3) To generate relationship matrix.

1) Product gene transcription (2)

The second stage of product gene transcription is from a relational matrix to the spatial predicate logic networks. Transformation rules are as follows: 
Perform the following actions: f_1= L(h),f_2= $\mathrm{R}(\mathrm{h}), \mathrm{f} \_\mathrm{i}=\mathrm{R}(\mathrm{h}-\mathrm{i}+2), \mathrm{i} \geqslant 3$, f_i represent feature I, L(h) represent left node of the $h$ layer, $R(h)$ represent right node of the $h$ layer, $\mathrm{R}(\mathrm{h}-\mathrm{i}+2)$ represent right node of the $\mathrm{h}-\mathrm{i}+2$ layer; the result is set the feature 1 at the left node of the highest layer; set the feature 2 at the right node of the highest layer; when $i \geqslant 3$, set the feature $i$ at the left node of the $h-i+2$ layer.

In biology, the process of "translation" corresponding to transcription. Biological gene translation is in the cytoplasm, messenger RNA as the template, under the action of enzymes and cofactors and energy, make the activated amino to protein polypeptide chain for a certain order process on the ribosome. The process of translation needs according to "code" rules. We regard three adjacent basic group on messenger RNA to determine an amino acid as a "codon". The codon can only decided an amino acid, an amino acid can be obtained by more than one codon.

The translation of product gene is also a very complicated process. The translation of product gene is form the spatial predicate logic network to product structure. Product gene translation process can be divided into two steps: (1) generate the multiple features of individual parts; (2) the feature makes up the part according to the spatial predicate logic network.

\section{CONCLUSION}

With the development of modern industry, intelligent design modeling has become the inevitable trend of product design. This paper introduces the concept of genes to the design of the product, put forward and set up product genetic model, aimed at apply the principle of gene expression to implement the product intelligent design, and strengthen the autonomy of product design, reduce artificial participation and related investment, improve the efficiency of product design, promote the development of product intelligent design. This paper first analyzes the problems existing in products genetic model, based on this, puts forward and establish the genetic model based on the feature of the products. On the basis of the definition of product gene structure, puts forward binary coding system of product gene and related operations. Then, based on product gene model, establish gene expression process model, product gene expression process is divided into two steps, transcription and translation, and transcription can be divided to two stages respectively. Finally, apply the theory of this paper to the engine products, to implement the intelligent design of the engine product, and has obtained the expected effect. At present, the field haven't reached maturity stage, further studies are needed.

\section{ACKNOWLEDGMENT}

The research work presented in this paper was supported by National Science and Technology Support Program: Electronic Devices Assembly High-speed Precision Robot and Automation Production Line R \& D and Industrialization (2015BAF10B01); Shanghai Science and Technology Commission Project: Multi-information visual recognition and 3D reconstruction key technology for industrial large-scale mechanical intelligent network control (14JC1402203).

\section{REFERENCES}

[1] Holland, J.H. Adaptation in Natural and Artificial Systems [M]. The University of Michigan Press, Ann Arbor, 1975

[2] Richard Myers. Edwin R Hancock. Empirical modeling of the genetic algorithms [J]. Evolutionary computation, 2001, 9(4):461-493.

[3] Chen Yong, Feng Peien, Lin Zhongqin. A genetics-based approach for the principle conceptual design of mechanical products [J]. The International Journal of Advanced Manufacturing Technology. 2005, 27: 225-233.

[4] Hao Yongtao, Qin Qin. Feature-function expression model and gene coding for products[J]. Proceedings of the 2009 Fifth International Conference on Natural Computation .2009, 6:160-166.

[5] Williams, H.T.P., Lenton, T.M. Artificial ecosystem selection for evolutionary optimization[J]. Advances in Artificial Life.2007:93-102

[6] Hao Yongtao, Shi Ming, Lou Diming. The research of the coupling between behavior and function based on behavior flow modeling[J]. International Journal of Digital Content Technology and its Applications. 2013,9(7): 236-45.

[7] Hao Yongtao, Tang Tao. The Research of Engine Modeling Based on the Petri Model of Behavior Flow[J]. 